\title{
On the Sum of Multiplicative Chi-Square-Lognormal Random Variables
}

\author{
Jan E. Tighe, Member, IEEE, and Tri T. Ha, Fellow, IEEE \\ Department of Electrical Engineering \\ Naval Postgraduate School, Monterey CA 93943-5000
}

\begin{abstract}
In order to analyze the performance of the DSCDMA cellular forward channel with convolutional encoding and soft-decision decoding operating in a Rayleigh-fading and lognormal-shadowing environment, we require the probability distribution function for a sum of $d$ multiplicative chisquare(with two degrees of freedom)-lognormal random variables. We approximate the sum using a multiplicative chisquare(with $2 d$ degrees of freedom)-lognormal random variable which is developed in this paper. We further investigate the usefulness of the approximation as it applies to the performance analysis of the DS-CDMA cellular forward channel.
\end{abstract}

\section{INTRODUCTION}

In [1] and a separate Globecom 2001 paper, we developed an upper bound on the probability of bit error $P_{e}$ for the forward channel of a DS-CDMA cellular system that employs forward error correction in the form of convolutional encoding and soft-decision decoding using the Viterbi algorithm in a mobile channel, which is subject to slow-flat Rayleigh fading and lognormal shadowing. This upper bound depends upon all possible first-event errors $P_{2}(d)$ that are a distance $d$ from the correct path. In order to calculate $P_{2}(d$ )for a specific distance $d$, we must first find the probability density function for the sum of $d$ chi-square(with 2 degrees of freedom)-lognormal random variables. The chi-square random variable comes from the transformation of the Rayleigh fading variable. If we let $M=R^{2} X$ represent our single chi-square(with 2 degrees of freedom)-lognormal random variable, then we can reasonably find the pdf for $M$, which we represent as $\mathrm{p}_{M}(\mathrm{~m})$. We let the random variable $Z_{d}$ represent a sum of $d$ independent identically distributed chisquare(with 2 degrees of freedom)-lognormal random variables as follows:

$$
Z_{d}=\sum_{l=1}^{d} R_{l}^{2} X_{/},
$$

where the $R_{/}$represent the Rayleigh fading random variables. The $X_{l}$ are lognormal random variables denoted by $X_{/} \sim \Lambda\left(0, \lambda \sigma_{d B}\right)$ where $\lambda=\ln 10 / 10$ and $\sigma_{d B}$ is the standard deviation of an underlying zero-mean Gaussian random variable (in decibels). We know that $Z_{d}$ has a probability distribution function that can only be determined by convolving $\mathrm{p}_{M}(\mathrm{~m})$ with itself $d$ times as follows:

$$
\mathrm{p}_{z_{d}}\left(z_{d}\right)=\underbrace{\mathrm{p}_{\mathrm{M}_{1}}\left(\mathrm{~m}_{1}\right) * \mathrm{p}_{\mathrm{M}_{2}}\left(\mathrm{~m}_{2}\right) * \ldots * \mathrm{p}_{\mathrm{M}_{d}}\left(\mathrm{~m}_{d}\right)}_{d} .
$$

Furthermore, for any particular convolutional encoder we require a series of $\mathrm{P}_{2}(d)$ and therefore require $\mathrm{p}_{z_{d}}\left(z_{d}\right)$ for $d=d_{\text {free }}, d_{\text {free }}+1, d_{\text {free }}+2, \ldots$ in order to calculate the upper bound on the probability of bit error $\mathrm{P}_{e}$ given by

$$
\mathrm{P}_{e} \leq \frac{1}{k} \sum_{d=d_{\text {free }}}^{\infty} \beta_{d} \mathrm{P}_{2}(d) \text {. }
$$

Even if we truncate the series using only the first 5 terms, we would need to perform $5 d_{\text {free }}+10$ convolutions, or integrations, to determine $\mathrm{P}_{e}$ for a particular operating scenario. In our analysis [1], we considered convolutional encoders with a code rate of $1 / 2$ and constraint lengths ranging from 7 to 9 . Typically, $d_{\text {free }}$ for such encoders range from 10 to 12 . Accordingly, for each operating scenario that we wish to consider, we would need to perform between 60 and 70 convolutions in order to calculate the upper bound on the probability of bit error $\mathrm{P}_{e}$ for that set of data. The computational cost in performing these integrations is too high a price to pay, which explains why analysis of coded DS-CDMA systems with soft-decision decoding that includes both fading and shadowing models has not previously been accomplished.

Our situation would be greatly improved if the channel model only considered the case of Rayleigh fading. In that case, our first-event error $\mathrm{P}_{2}(d)$ would depend only upon a sum of chi-square random variables. We know that the sum of $d$ chi-square random variables with two degrees of freedom is precisely another chi-square random variable with $2 d$ degrees of freedom [2]. Accordingly, the conditioning can be removed from $\mathrm{P}_{2}(d)$ analytically. Similarly, if we consider the case of lognormal shadowing only (no Rayleigh fading), our first-event error $\mathrm{P}_{2}(d)$ depends only upon a sum of $d$ independent lognormal random variables. Since the sum of lognormal random variables appears in many important communications, radar, and antennae problems, considerable effort has been given in finding its distribution function [3]-[6]. While no closed form solution for the distribution function has been found, there is agreement that the sum of lognormal random variables can be approximated as another lognormal random variable. Accordingly, for the case of strictly lognormal shadowing, we can remove the conditioning of $\mathrm{P}_{2}(d)$ on the sum of lognormal random variables by approximating the sum as another lognormal random variable. Consequently, we can solve for the upper 
bound on the probability of bit error for lognormal shadowing only.

In summary, the distribution of the sum of $d$ chi-square random variables with 2 degrees of freedom is chi-square with $2 d$ degrees of freedom. Furthermore, the distribution of the sum of lognormal random variables can be approximated as lognormal. In light of these two facts, we approximate our sum of $d$ chi-square(with 2 degrees of freedom)-lognormal random variables as a multiplicative chi-square(with $2 d$ degrees of freedom)-lognormal random variable.

\section{DeFINING THE MODEL}

In order to approximate $Z_{d}$ as a chi-square(with $2 d$ degrees of freedom)-lognormal random variable, we first represent our model $\widetilde{Z_{d}}$ in terms of its two factors defined by

$$
\widetilde{Z_{d}}=\mathrm{Y}_{2 d} \mathrm{X}_{Z}
$$

where $Y_{2 d}$ is a chi-square random variable with $2 d$ degrees of freedom, whose distribution is completely specified based on the underlying Rayleigh random variable, i.e., $\underset{\text { by }}{Y_{2 d}}=\sum_{d} R^{2}$. Accordingly, the moments of $Y_{2 d}$ are defined

$$
\begin{aligned}
& \mathrm{E}\left\{\mathrm{Y}_{2 d}\right\}=d \mathrm{E}\left\{R^{2}\right\}=d, \\
& \mathrm{E}\left\{\mathrm{Y}_{2 d}^{2}\right\}=d+d^{2},
\end{aligned}
$$

and $\mathbf{V} \operatorname{ar}\left\{\mathbf{Y}_{2 d}\right\}=d$,

where we have normalized $\mathrm{E}\left\{R^{2}\right\}=1$. Furthermore, $X_{Z} \sim \Lambda\left(\mu_{Z}, \sigma_{Z}\right)$ is the lognormal component of $\widetilde{Z_{d}}$, where the model parameters $\mu_{Z}$ and $\sigma_{Z}$ are yet to be developed. The moments of $X_{Z}$ are given by

$$
\begin{aligned}
& \mathrm{E}\left\{\mathrm{X}_{Z}\right\}=e^{\mu_{Z}+\frac{\sigma_{Z}^{2}}{2}}, \\
& \mathrm{E}\left\{\mathrm{X}_{Z}\right\}=e^{2 \mu_{Z}+2 \sigma_{Z}^{2}},
\end{aligned}
$$

$$
\text { and } \mathbf{V} \operatorname{ar}\left\{\mathrm{X}_{Z}\right\}=e^{2 \mu_{Z}+\sigma_{Z}^{2}}\left(e^{\sigma_{Z}^{2}}-1\right) \text {. }
$$

We develop the moments for our model by assuming that $Y_{2 d}$ and $X_{Z}$ are independent, from which we obtain

$$
\begin{aligned}
\mathrm{E}\left\{\widetilde{Z_{d}}\right\} & =d \exp \left(\mu_{Z}+\frac{\sigma_{Z}^{2}}{2}\right), \\
\mathrm{E}\left\{\widetilde{Z_{d}}{ }^{2}\right\} & =\left(d+d^{2}\right) \exp \left(2 \mu_{Z}+2 \sigma_{Z}^{2}\right), \\
\text { and } \mathbf{V} \boldsymbol{a}\left\{\widetilde{Z_{d}}\right\} & =d e^{2 \mu_{Z}+\sigma_{Z}^{2}}\left[(1+d) e^{\sigma_{Z}^{2}}-d\right] .
\end{aligned}
$$

The remaining step in approximating $Z_{d}$ as chi-square(with $2 d$ degrees freedom)-lognormal is in determining the model parameters $\mu_{Z}$ and $\sigma_{Z}^{2}$ for $X_{Z}$. We develop these two parameters by relating the mean and variance of our model $\widetilde{Z_{d}}$ with a scaled version of mean and variance of the original chi-square2-lognormal random variable, $R^{2} X$. We equated the mean of our model in (7) with $d$ times the mean of $R^{2} X$ scaled by a factor of $\mathrm{g}_{1}$ and solve for the parameter $\mu_{Z}$ as follows:

$$
\mu_{Z}=\frac{\left(\sigma_{X}^{2}-\sigma_{Z}^{2}\right)}{2}+\ln \left(g_{1}\right)
$$

where $\sigma_{X}^{2}=\lambda^{2} \sigma_{d B}^{2}$ is the parameter from our original lognormal shadowing random variable, $X \sim \Lambda\left(0, \lambda \sigma_{d B}\right)$. In order to define the parameter $\sigma_{Z}^{2}$, we equated the variance of our model with $d$ times the variance of $R^{2} X$ scaled by a factor of $\mathrm{g}_{2}$ and solved for $\sigma_{Z}^{2}$ as follows:

$$
\sigma_{Z}^{2}=\ln \left[\frac{g_{2}}{g_{1}^{2}}\left(2 e^{\sigma_{X}^{2}}-1\right)+d\right]-\ln (1+d) .
$$

The scaling factors $\mathrm{g}_{1}$ and $\mathrm{g}_{2}$ of the mean and variance, respectively, vary with $\sigma_{d B}$ and are chosen such that the CDF of our model $\widetilde{Z_{d}}$ best matches the CDF of $Z_{d}$ for $d=8,9, \ldots, 16$. Table 4.1 depicts the values of $g_{1}$ and $g_{2}$ for each $\sigma_{d B}$ as determined by simulation.

Table 1

\begin{tabular}{|l|l|l|}
\hline \multicolumn{3}{|c|}{ Values of $\mathrm{g}_{1}$ and $\mathrm{g}_{2}$ for $\widetilde{z_{d}}$} \\
\hline$\sigma_{d B}$ & $\mathrm{~g}_{1}$ & $\mathrm{~g}_{2}$ \\
\hline 2 & 1 & .9875 \\
\hline 3 & 1 & .9625 \\
\hline 4 & 1 & .9125 \\
\hline 5 & 1 & .8125 \\
\hline 6 & .9375 & .4875 \\
\hline 7 & .9125 & .3375 \\
\hline 8 & .8625 & .2 \\
\hline 9 & .8 & .1 \\
\hline
\end{tabular}

With the model parameters completely specified, we know that the pdf of $\widetilde{Z_{d}}$ can be written as a product of its independent component elements; namely $Y_{2 d}$ and $X_{Z}$ as

$$
p_{\widetilde{Z_{d}}}(\mathrm{y}, \mathrm{x})=\mathrm{p}_{\mathrm{r}_{2 d}}(\mathrm{y}) \mathrm{p}_{\mathrm{x}}(\mathrm{x})
$$

Alternatively, we can develop the pdf of $\widetilde{Z_{d}}$ as a function of $z$ using the marginal densities of its components given by

$\mathrm{p}_{\widetilde{Z_{d}}}(z)=\frac{z^{d-1}}{\sqrt{2 \pi} \sigma_{z} \Gamma(d)} \int_{0}^{\infty} \frac{1}{\mathrm{x}^{d+1}} \exp \left(\frac{-z}{\mathrm{x}}-\frac{\left(\ln \mathrm{x}-\mu_{z}\right)^{2}}{2 \sigma_{z}^{2}}\right) d \mathrm{x}$,

where $\mu_{Z}$ and $\sigma_{Z}^{2}$ are defined by (8) and (9), respectively.

We have developed a distribution for the chi-square(with $2 d$ degrees freedom)-lognormal random variable $\bar{Z}_{d}$, which approximates the distribution of a sum of $d$ chi-square(with 2 degrees freedom)-lognormal random variables. 


\section{TESTING THE MODEL}

In this section we will explore the usefulness of our model as it applies to bit error analysis of DS-CDMA with FEC. As an example, we will consider the case of $\sigma_{d B}=7$ with rate $1 / 2$ convolution encoding with $v=8$ and approximate the probability of bit error using the first five terms of the union bound from (3). Accordingly, we will examine our model's ability to approximate $P_{2}(d)$ for $d=10$ through 14 , since these are the key parameters when employing rate $1 / 2$ convolutional encoding with constraint lengths 7 and 8.

Figs. 1 through 3 show histograms for $Z_{d}$ with $\sigma_{d B}=7$ and $\mathrm{E}\left\{R^{2}\right\}=1$, which were formed by generating $d$ sets of multiplicative chi-square(with 2 degrees freedom)-lognormal distributed data consisting of 400,000 independent samples per set and summing over the $d$ sets. We have over-plotted the histogram with the pdf of our model $\widetilde{Z_{d}}$.

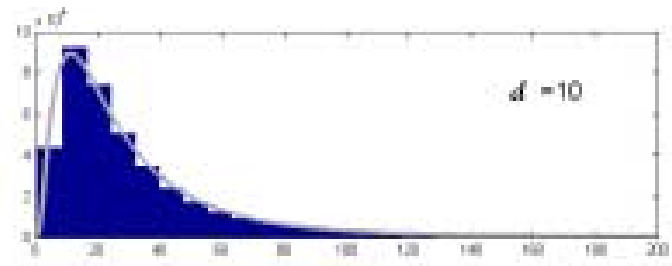

Fig.1 Histogram of $Z_{10}$ and the PDF for $\widetilde{Z_{10}}$ for $\sigma_{d B}=7$

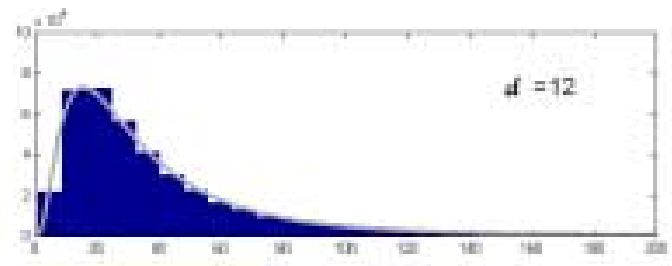

Fig.2 Histogram of $Z_{12}$ and the PDF for $\widetilde{Z_{12}}$ for $\sigma_{d B}=7$

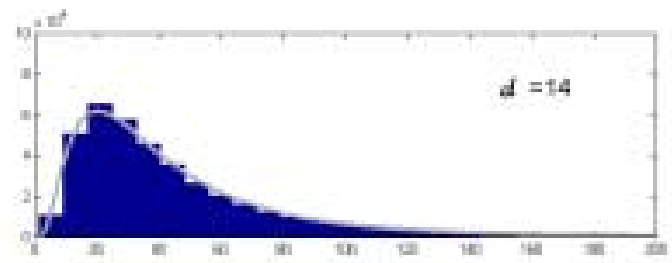

Fig. 3 Histogram of $Z_{14}$ and the PDF for $\widetilde{Z_{14}}$ for $\sigma_{d B}=7$

We know that the distribution of $Z_{d}$ is not chi-square(with $2 d$ degrees freedom)-lognormal as approximated by $\widetilde{Z_{d}}$. The real question is whether $\widetilde{Z_{d}}$ can well approximate $Z_{d}$ in calculating the first-event error probability. In order to determine this answer, we evaluate $P_{2}(d)$ as developed in [1] by simulation and compare with the result predicted by our model. We simulate the integral by generating $d$ independent samples from the multiplicative chi-square(with 2 degrees freedom)-lognormal distribution and summing them to form one realization $\rho_{1}$ of $\mathrm{P}_{2}(d)$. We repeat this process 100,000 times and form our point estimate for $\mathrm{P}_{2}(d)$ as follows:

$$
\bar{\rho}=\frac{1}{10^{5}} \sum_{i=1}^{10^{5}} \rho_{i} .
$$

Figs. 4 through 6 show the point estimates for the simulated $\mathrm{P}_{2}(d)$ as compared with its approximation using our model for the case of $\sigma_{d B}=7$ with 10 users per adjacent cell and $d=10$ through 14 . In these cases, our model well approximates the simulated result for $\mathrm{P}_{2}(d)$.

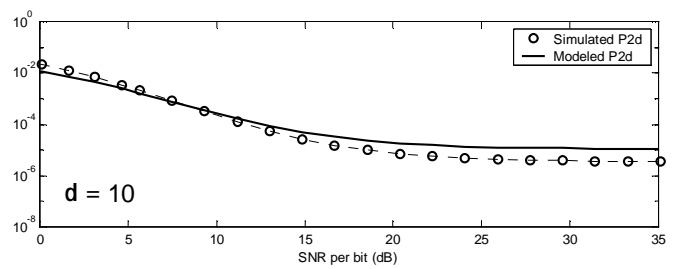

Fig. 4 First Event Error Probability $\mathrm{P}_{2}(10)$ for $\sigma_{d B}=7$ with 10 users/cell

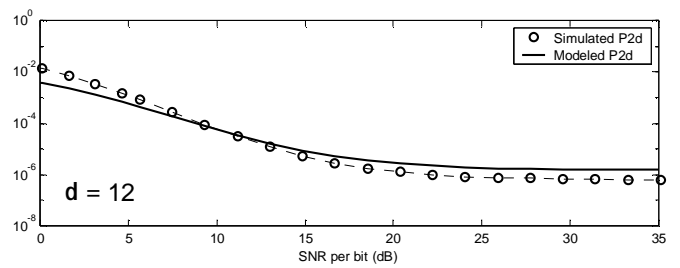

Fig. 5 First Event Error Probability $\mathrm{P}_{2}(12)$ for $\sigma_{d B}=7$ with 10 users/cell

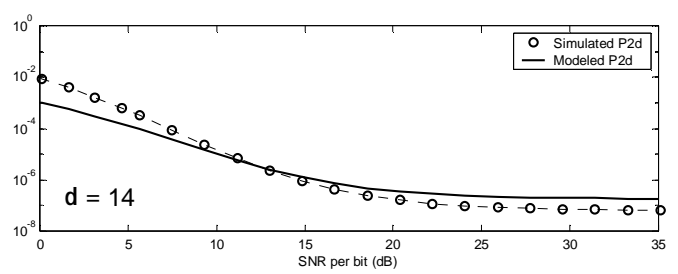

Fig. 6 First Event Error Probability $\mathrm{P}_{2}(14)$ for $\sigma_{d B}=7$ with 10 users/cell

We incorporate these results into (3) to determine the probability of bit error using the first five terms in the union bound. For a rate $1 / 2$ convolutional encoder with constraint length of $v=8$, we obtain a modeled and simulated probability of bit error as shown in Fig. 7.

By selecting a constraint length of $v=8$ as an example, we incorporate the modeled and simulated results of $\mathrm{P}_{2}(d)$ for $d=10$ through $14, d_{\text {free }}=10$ and $\beta_{10}=2, \beta_{11}=22$, $\beta_{12}=60, \beta_{13}=148, \beta_{14}=340$. In the case of 10 users per cell, small differences between the modeled and simulated $P_{2}(d)$, as shown in Figs. 3 and 4, grow larger when their weighted sums are applied in the union bound (3), although the differences are still quite small (on the order of $10^{-4}$ ). Nevertheless, these differences do not outweigh the usefulness of our model in analyzing the performance of DS- 


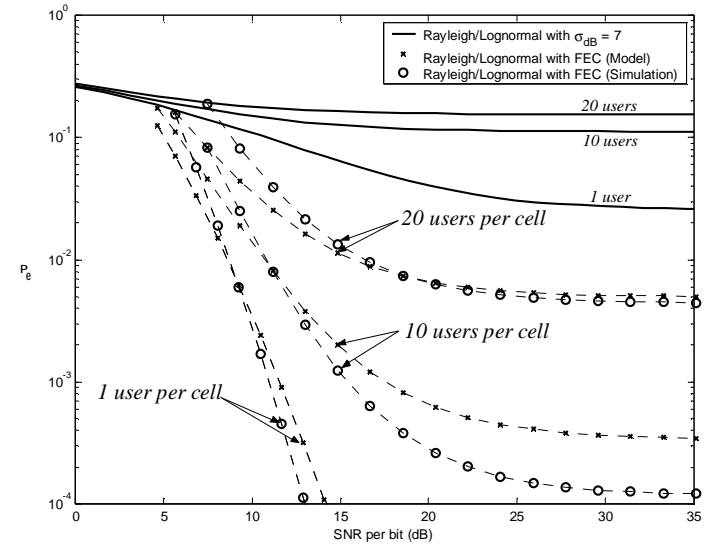

Fig. 7 Probability of Bit Error for DS-CDMA in Rayleigh-Lognormal Channel $\left(\sigma_{d B}=7\right)$ using a Rate $1 / 2$ Convolution Encoder $v=8$

CDMA cellular systems. In particular, the model closely matches the simulated result in the range from 10 to $15 \mathrm{~dB}$, which is a practical range in which DS-CDMA cellular systems operate. Furthermore, the differences in the modeled and simulated results appear most obvious when the interference floor is in a range between $10^{-3}$ and $10^{-7}$, where even the slightest differences are magnified due to the logarithmic presentation of results. Fig. 8 reflects the modeled versus simulated results in the case of $\sigma_{d B}=4$ and where we have included antennae sectoring in the analysis. Modeling results for the full range of the model, $2 \leq \sigma_{d B} \leq 9$, can be found in [1].

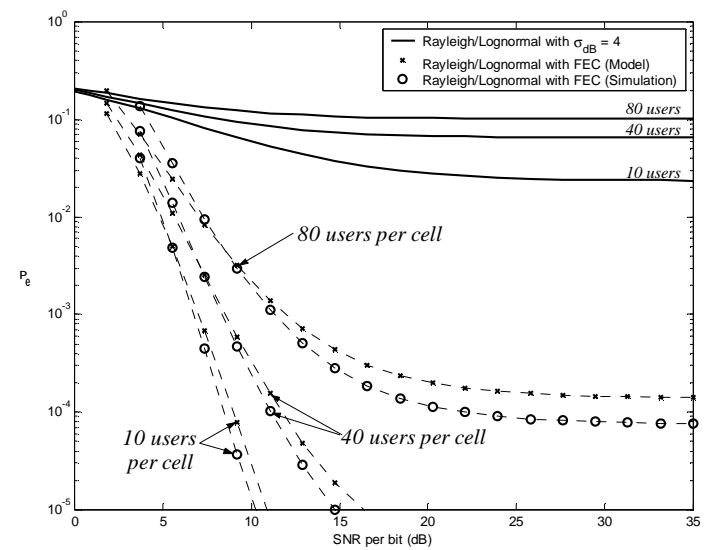

Fig. 8 Probability of Bit Error for DS-CDMA with Rayleigh Fading and Lognormal Shadowing $\left(\sigma_{d B}=4\right)$ using $120^{\circ}$ Sectoring $\left(\mathrm{R}_{\mathrm{cc}}=1 / 2\right.$ and $\left.v=8\right)$

\section{CONCLUSIONS}

The multiplicative chi-square(with $2 d$ degrees of freedom)lognormal random variable can well approximate the distribution for a sum of multiplicative chi-square(with 2 degrees of freedom)-lognormal random variables. This approximation makes the performance analysis of the DS-
CDMA forward channel with forward error correction and soft-decision decoding in Rayleigh-fading and lognormalshadowing environments more palpable. Furthermore, the model may be suitable in other applications such as maximal ratio combining and antenna diversity models. Our model is specifically optimized for sums of between 10 and 15 random variables as required for the convolutional encoder employed. In applications with sums fewer than 10 or greater than 15 , alternative values of $\mathrm{g}_{1}$ and $\mathrm{g}_{2}$ may need to be determined to best approximate the sum.

\section{REFERENCES}

[1] J. E. Tighe, 'Modeling and Analysis of Cellular CDMA Forward Channel," Ph.D. Dissertation, Department of Electrical and Computer Engineering, Naval Post Graduate School, Monterey, CA, Mar. 2001.

[2] J. G. Proakis, Digital Communications, Boston, Massachusetts. WCB/McGraw-Hill, 1993.

[3] S. C. Schwartz and Y. S. Yeh, "On the Distribution Function and Moments of Power Sums with Log-Normal Components," Bell System Tech. Journ., vol. 61, no. 7, pp. 1441-1462, Sep. 1982.

[4] D. Schleher, "Generalized Gram-Charlier Series with Application to the Sum of Lognormal Variates," IEEE Trans. on Inform. Theory, pp. 275-280, Mar. 1977.

[5] N. C. Beaulieu, A. A. Abu-Dayya, and P. J. McLane, "Estimating the Distribution of a Sum of Independent Lognormal Variables," Queen's University Tech. Report \#9401, Jan. 1994.

[6] A. A. Abu-Dayya, N. C. Beaulieu, "Comparison of Methods of Computing Lognormal Sum Distributions and Outages for Digital Wireless Applications," in Conf. Rec. SUPERCOMM '94, vol. 3, pp. 1270-1275, May 1994. 